\title{
CONSTRUCTION AND TESTING OF AN 11.4 GHz DIELECTRIC STRUCTURE BASED TRAVELLING WAVE ACCELERATOR
}

\author{
Peng Zou, Wei Gai, R. Konecny and T. Wong \\ Argonne National Laboratory, Argonne, IL 6039, USA
}

Abstract: One major challenge in constructing a dielectric loaded travelling wave accelerator powered by an external rf power source is the difficulty in achieving effcient coupling. In this paper, we report that we have achieved high effiency broadband coupling by using a combination of a tapered dielectric section and a carefully adjusted coupling slot. We are currently constructing an $11.4 \mathrm{GHz}$ accelerator structure loaded with a permitivity $=20$ dielectric. Bench testing has demonstrated a coupling efficiency in excess of $95 \%$ with bandwidth of $600 \mathrm{MHz}$. The final setup will be tested at high power at SLAC using an X-band klystron rf source.

\section{I . Introduction:}

The proposed use of if driven dielectric based structures for particle acceleration can be traced to the early 50's [1]. Since then, numerous studies have examined the use of dielectric materials in accelerating structures[2,3]. Advantages and potential problems of using dielectric material are discussed in the references. More that recent development of high dielectric constant $(\varepsilon \sim 20-40)$, low loss materials $(\mathrm{Q} \sim 10,000-40,000)$ warrant a new look at the idea $[4$, transtech].

One faces a challenging problem when building an actual dielectric accelerator because outer diameter of the dielectric is much smaller than the rectangular waveguide which couples the external rf. Therefore, realising impedance matching becomes a difficult tasks. There is also no technical references treating this subject. We found that by using a combination of side coupled slots and a tapered dielectric near the coupling slots, one can efficiently couple the $\mathrm{rf}$ from the rectangular waveguide to the dielectric waveguide. In the prototype $11.4 \mathrm{GHz}$ dielectric loaded accelerator as shown in Figure 1, we have achieved $>95 \%$ power coupling.

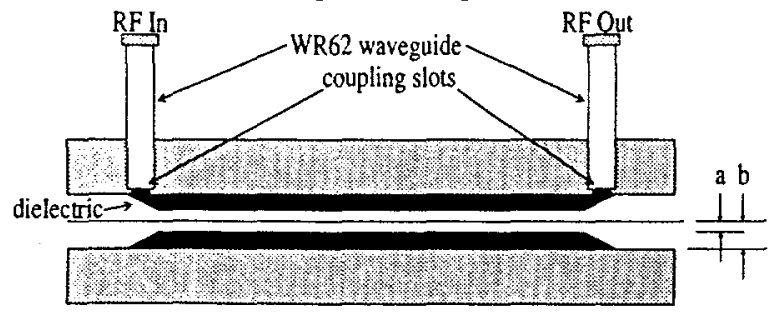

Figure 1. Schematic diagram of a dielelectric loaded travelling wave accelerator.

To be presented at the 1999 Particle Accelerator Conference, (PAC'99), New York, New York, March 29 to April 02, 1999.

\section{A Travelling Wave Dielectric Loaded Accelerating Structure Basics}

The dielectric travelling wave accelerator has a simple geometry. Considering a cylindrical structure partially filled with dielectric material $(\varepsilon)$ with inner radius $a$, outer radius $b$ and conducting wall on the outside. There are also two ports on the side for RF coupling purposes, as shown in Figure 1. The axial electric fields inside the structure can be solved for exactly as

$$
\begin{aligned}
& E_{z}{ }^{(1)}=E_{0} I_{0}(k r) e^{i\left(k_{z} z-\omega t\right)} \\
& E_{z}{ }^{(2)}=\left[B_{1} J_{0}\left(s_{1} r\right)+D_{1} N\left(s_{1} r\right)\right] e^{i(k z-\omega t)}
\end{aligned}
$$

Here $E_{0}, B_{0}$ and $D_{1}$ are the field amplitudes in the region 0 (vacuum) and 1 (dielectric) respectively and are related by boundary conditions and

$$
\begin{aligned}
& k^{2}=\frac{\omega^{2}}{c^{2}}\left(1-\beta^{2}\right) \\
& s^{2}=\frac{\omega^{2}}{v^{2}}\left(\beta^{2} \varepsilon-1\right)
\end{aligned}
$$

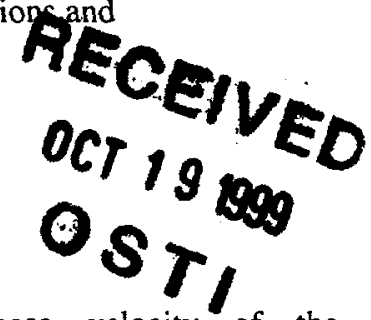

where $\beta c=\nu=\omega / k$ is phase velocity of the wave travelling inside the tube: $\beta$ determines the synchronism of the wave and the accelerated particles. By properly choosing $a, b$ and $\varepsilon$, one can adjust the phase velocity accordingly. Thus this proposed scheme works not only for acceleration of electrons which typically has phase velocity $\sim c$, but also for low phase velocity particle acceleration, such as heavy ions. The transverse electric field can be written as

$E_{r}=\frac{i}{\frac{\omega}{\nu}\left(\beta^{2} \varepsilon-1\right)} \frac{\partial E_{z}}{\partial r}$ $\therefore$

and the magnetic field $H_{\phi}=\varepsilon E_{r}$ everywhere inside the tube. By matching the boundary conditions at $a$ and $b$ ( $E_{z}$ and $D_{r}$ continous), all the field components can be calculated accordingly.

The stored energy per unit length $U$ in the tube is the sum of contributions from both vacuum and dielectric regions, and can be expressed as

The submitted manuscript has been create. by the University of Chicago as Operator c Argonne National Laboratory ("Argonne"
under Contract No. W-31-109-ENG-38 wit the U.S. Department of Energy. The U.S Government retains for itself, and others ac ing on its behalf, a paid-up, nonexclusive irrevocable worldwide license in said articl to reproduce, prepare derivative works, dis tribute copies to the public, and perform puk licly and display publicly, by or on behalf c the Government. 


\section{DISCLAIMER}

This report was prepared as an account of work sponsored by an agency of the United States Government. Neither the United States Government nor any agency thereof, nor any of their employees, make any warranty, express or implied, or assumes any legal liability or responsibility for the accuracy, completeness, or usefulness of any information, apparatus, product, or process disclosed, or represents that its use would not infringe privately owned rights. Reference herein to any specific commercial product, process, or service by trade name, trademark, manufacturer, or otherwise does not necessarily constitute or imply its endorsement, recommendation, or favoring by the United States Government or any agency thereof. The views and opinions of authors expressed herein do not necessarily state or reflect those of the United States Government or any agency thereof. 


\section{DISCLAIMER}

Portions of this document may be illegible in electronic image products. Images are produced from the best available original document. 


$$
\begin{aligned}
U & =\frac{1}{2} \sum_{0,1} \pi \int\left(\varepsilon \varepsilon_{0} E^{2}+\mu_{0} B^{2}\right) r d r \\
& =E_{0}{ }^{2} u
\end{aligned}
$$

where $u$ is a geometric factor which depends solely on the structure geometry and dielectric constant. For a given RF power, the axial electric field in the center region of the tube can be expressed as

$$
E_{0}=\left[\frac{P}{u \beta_{g} c}\right]^{\frac{1}{2}}
$$

where $\beta_{z}$ is the group velocity. The dielectric loss plus wall loss per unit length is then found from

$$
\eta=\frac{2 \pi \delta U_{o u t}}{v_{g}\left(U_{o u t}+U_{i n)}\right.}+\frac{R_{s} \oint_{\text {Wall }} d s<H^{2}>}{\omega U}
$$

The electric fields in the vacuum region described by equation 1 and 3 have very interesting chraracteristics. When $k \rightarrow 0$, i.e., the phase velocity of the wave is $\mathrm{c}, E_{z}$ is constant in $r$. This implies that there are no focusing and de-focusing forces for a relativistics particle travelling inside the vacuum chamber. This is critical for emittance preservation in the linacs, particularly for high brightness electron gun development.

\section{Construction and Bench Testing of the $11.4 \mathrm{GHz}$ Structures.}

We have developed a design for an X-band structure (11.4 Ghz) using the parameters given in table I. Choice of the dielectric is $\mathrm{MgCaTi}$ compound which has dielectric constant of 20 . And this material can be readily obtained from commercial vendors. The group velocity for the NLC design is in the $0.03 c \sim 0.05 c$ range [5]. Thus dielectric loaded structure is having a comparable shunt impedence and group velocity to a conventional X-band structure as indicated in the table below. As shown above, one of the interesting characteristics of this structure is that the frequency of the HEM11 mode (first deflection mode) is lower than that of the acceleration mode. Because the deflection force is a function of $\sin (k z)$, this implies very different and improved conditions for the single bunch BBU problem compared to conventional structures where the HEM11 is always higher in frequency than the accelerating TM01 mode.

Table : Dimensions and physical properties of the 11.4 $\mathrm{GHz}$ dielectric tube

$\begin{array}{ll}\text { Material } & \text { MgCaTi } \\ \varepsilon \text { (diele. const.) } & 20 \\ \text { Tapered Angle } & 8^{\circ} \\ \text { Loss tangent } \delta & 10^{4} \\ \text { Inner Radius a } & 0.3 \mathrm{~cm}\end{array}$

$\begin{array}{ll}\text { Outer Radius b } & 0.456 \mathrm{~cm} \\ \text { HEM } 11 & 9.96 \mathrm{~Hz} \\ \text { group velocity } & 0.057 \mathrm{c} \\ \text { Attenuation } & 4 \mathrm{~dB} / \mathrm{m} \\ \text { Power needed }(10 \mathrm{MV} / \mathrm{m}) & 2.6 \mathrm{MW}\end{array}$

The RF coupling scheme we used here is similiar to the side coupled method used for conventional diskwasher RF cavities. Impedance matching of the coupling slotsis more difficult in the high $\varepsilon$ dielectric case because the outer radius of the dielectric tube is much smaller than the waveguide.

Basically speaking, one would like to obtain maximum RF transmission through the two coupling slots. In order to achive high efficiency coupling, the dielectric tube near the coupling slots is tapered. The tapered angle was chosen to be $8^{\circ}$ for initial convenience. This tapered section serves as a broad band quarter wave transformer for impedance matching. No other angles were tested, but it is not expected that the taper angle is critical. The detailed configuration of the tapered dielectric structure and coupling slots are shown in Figure 1.

A $25 \mathrm{~cm}$ long prototype structure was constructed with the parameters in table 1 . The dielectric materials was obtained from Trans Tech. The coupling slot dimensions are $4.7 \mathrm{~mm} \times 5.69 \mathrm{~mm}$. By careful adjustment of the coupling slots and monitoring the S-parameters using an HP8510C network analyzer, we have achieved a reflection coefficeint $\mathrm{S} 11<-13 \mathrm{~dB}$ and transmission $\mathrm{S} 21>-1.5 \mathrm{~dB}$ @ $11.43 \mathrm{GHz}$ as shown in Figures 2 and 3 respectively.

We plan to continue engineering studies of this accelerating structure with improved RF coupling and mechanical fixture to allow operation in vacuum, eventually leading to a high power test at SLAC of a demonstration accelerator section to resolve practical issues such as breakdown voltage, thermal heating etc. With $100 \mathrm{MW}$ power, we can test this structure at a 60 $\mathrm{MV} / \mathrm{m}$

gradient.

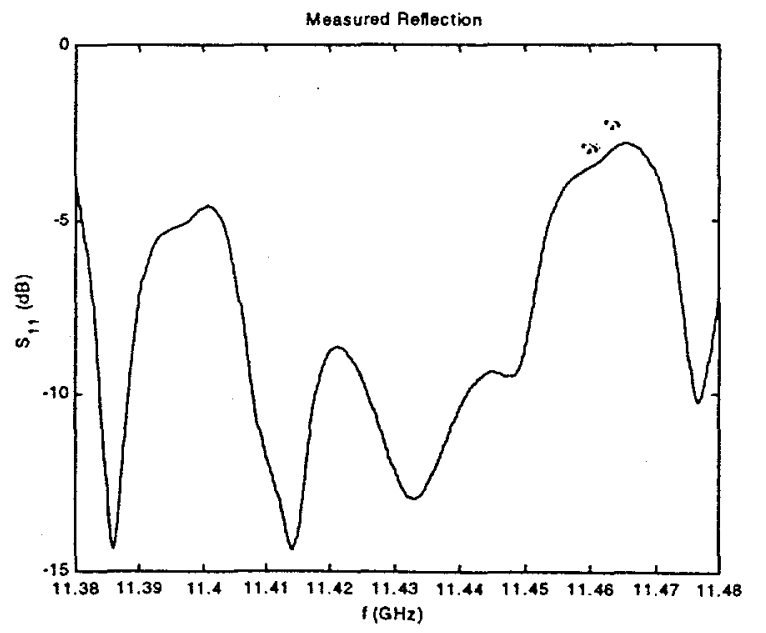

Figure 2. S11 for the optimally coupled waveguide results. 


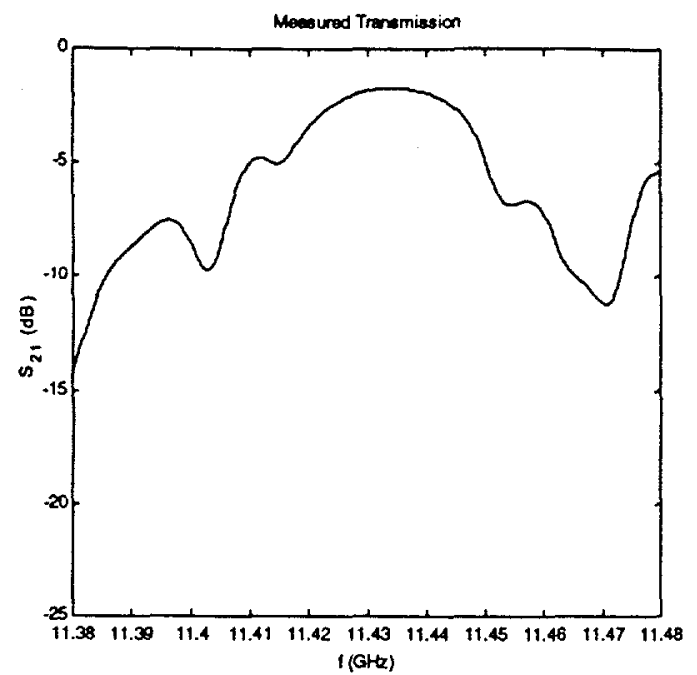

Figure 3. S21 between two optimally coupled 2 ports

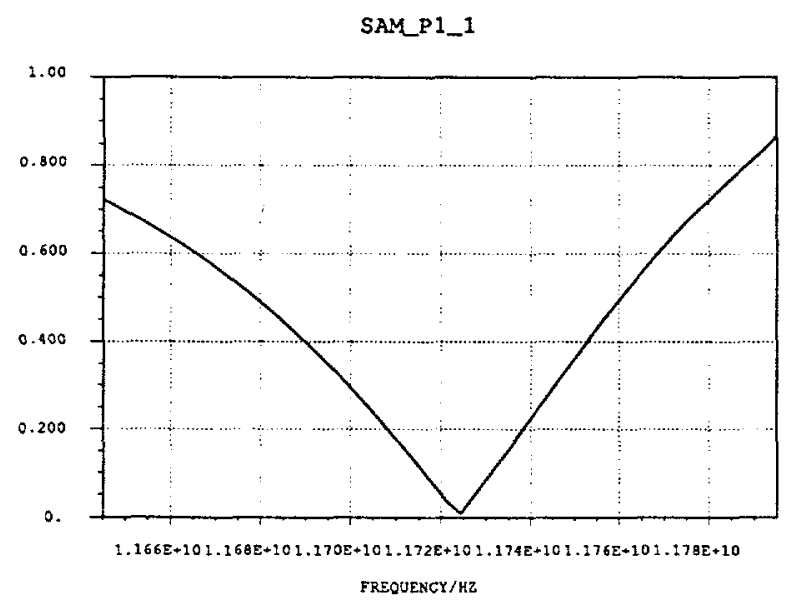

Figure 4. Calculated S11 parameter using MAFIA At $11.7 \mathrm{GHz}$, the $\mathrm{S} 11$ is almost 0 .

\section{Numerical simulations of the coupling port using MAFIA}

In order to verify the coupling method developed experimentally, we have used MAFIA [6] to simulate the parameters described in the last section. As expected, the simulation process was tedious and time consuming. Due to the relatively small size of the coupling slot, special attention has to be given for mesh size in the neighborhood of the slot.

Figure 4 shows the calculated S11, although it is peaked at $11.7 \mathrm{GHz}$ for the given geometry, it does give qualitative agreement with the bench top measurement. In the calculation, with assumption of no dielectric loss and no wall loss, we have achieved $S 21$ of near 1.0 as shown in Figure 5. In comparison with $-1.6 \mathrm{~dB}$ measurement in Figure 3 where the wall losses dominated.

\section{Summary}

In summary, we have constructed and studied a prototype $11.4 \mathrm{GHz}$ dielectric loaded waveguide. Careful engineering considerations were implemented. We have achieved efficient coupling from port to port. A demonstration accelerator for high power test has been designed and is under construction. Our goal is to achieve $50-100 \mathrm{MV} / \mathrm{m}$ so it can be used as a viable alternative for the accelerating structures. Some pratical issues concerning high power breakdown and thermal heating will be answered through careful experimental studies and new materials development.

This work is supported by DOE, High Energy Physics Division under contract No. W-31-109-ENG-38.

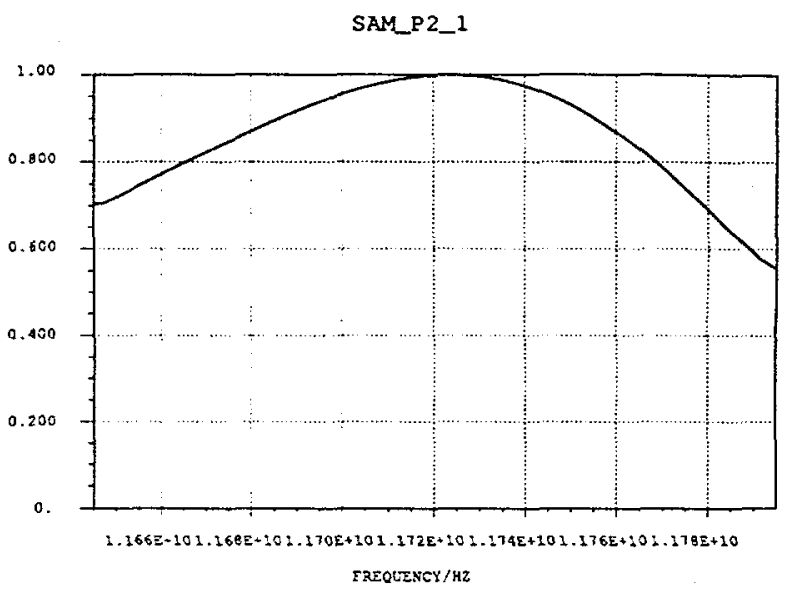

Figure 6. Calculated S21 corresponding to the parameters in the Figure 5. Almost perfect coupling was achieved here.

\section{References:}

1. G. Flesher and G. Cohn, AIEE Transactions, 70,887 (1951)

2. B. Zhang et. al., in Proceedings of 7th Advanced Accelerator Worksop, AIP Proceedings No. 398, p. 618(1996).

3. W. Gai, R. Konecny and J. Simpson in Proceedings of 1997 Particle Accelerator Conference, p. 636-638, Vancourver, May 1997

4. Trans-Tech., Catalog, 5520 Adamstown, Adamstow, MD 21710T.

5. SLAC repor 474,1996

6. MAFIA Version 4.0, Gesellschaft fur ComputerSimulationstechnik, Lauteschlagerstrabe 38, D-64289, Darmstadt. 